\title{
SOLID STATE QUANTUM INTERFEROMETERS (WITH AND WITHOUT THE WARTS)*
}

\author{
S. WASHBURN \\ Department of Physics and Astronomy \\ The University of North Carolina at Chapel Hill \\ Chapel Hill, NC 27599-3255, USA
}

\begin{abstract}
A brief review of quantum interference in small conductors is followed by a discussion of quantum interference in ballistic conductors. The ballistic conductors are characterized by conservation of the momentum vector and this leads to a simple model for the details of the conductance of the system. In contrast, for diffusive conductors one can only predict the statistical behavior of the interference contributions to the conductance. The response of the ballistic interferometers is governed by semiclassical physics of the electron momentum states (i.e. the modes) in the quantum conductor. Under circumstances of reduced backscattering of the modes, the interference pattern appears to imitate that from a simple optical interferometer. New results from ballistic interferometers are reviewed briefly.
\end{abstract}

PACS numbers: $73.20 . \mathrm{Dx}$

The advent some years ago of quantum interference experiments led to a small revolution in the understanding of transport of electrons in the solid state [1]. First [2] in samples of size $L$ much greater than the quantum coherence length $L_{\varphi}$ of the carriers and later [3] in samples of size $L \approx L_{\varphi}$, quantum interference was shown to be a significant perturbation on the average (classical) conductance. The quantum corrections to the average conductance arise through the interference of coherently propagating carriers, which contribution was ignored in the calculations of the average (Drude) conductance. Carrier excitations from the Fermi sea propagate for some distance and are scattered elastically by static impurities (lattice defects) along the way. The probability for a carrier to propagate from a certain point to another is given by the usual sum over all Feynman paths $W=\left|\sum_{n} Q_{n}\right|^{2}$ where $Q_{n}$ is a complex amplitude to propagate along the $n$-th trajectory between the two points. The total conductance of a conductor with two ports is $G=\left(e^{2} / h\right) \sum_{m} W_{m}$, where $W_{m}$ is the transmission probability for the $m$-th mode in the conductor. The excitations are annihilated by inelastic scattering from dynamic excitations such as other carriers, phonons, spins, etc. [1]. At low

*This work was supported by IBM, MCNC and The University of North Carolina at Chapel Hill. 
temperatures, there is very little thermal energy available to support populations of dynamic excitations, and so the length scale $L_{\text {in }}$ over which the excitations propagate can be rather long, typically reaching several microns below a temperature of $T=1 \mathrm{~K}$. In garden variety metals, the mean free path length $l$, which is the length scale for elastic scattering from impurities, is much smaller - rarely exceeding a few tens of nanometers. This means that in the metal, the excitation propagates along a random walk undergoing many elastic collisions along the way [2]. In contrast, in clean samples, where there is very little static disorder, the elastic mean free path can be many microns or even millimeters. The cleanest materials are single crystals of metals in which mean free paths of centimeters are found [4], but in clean semiconductors, it is possible to make circuits in which all circuit dimensions are smaller than the physically important length scales $l$ and $L_{\varphi}[5]$.

Results from quantum transport experiments on both clean and disordered samples clearly demonstrate the inadequacy of the simple average behavior of the transport coefficients. In spite of the great number of independent transport channels through even the smallest available conductors, and in spite of the vast number of impurities in some of the metals samples, the fluctuations in conductance with rearrangement of the impurities do not shrink to zero [6]. Instead the amount of fluctuation approaches a constant even for arbitrarily large samples (so long as the whole region is within the phase coherence length). It approaches a constant rather than vanishing as the sample size increases because of correlations among the trajectories through the impurity potential [7]. The correlation is strong for trajectories of excitations that are proximate in energy, and the contributions of such trajectories add together "in phase" and reinforce each other's contribution to the conductance [8], so that the effective number of trajectories is much less than the simple classical estimate $L^{2} k_{\mathrm{F}}^{2}$ ( $L$ is the sample dimension and $k_{\mathrm{F}}$ is the Fermi wave number), which for a $50 \mathrm{~nm} \times 50 \mathrm{~nm} \mathrm{Au}$ wire is $\approx 10^{6}$. For excitation energies that are disparate in energy, the trajectories are not correlated and so their contributions to the conductance average together as random quantities [9]. The lack of reinforcement in the contributions of disparate trajectories is a source of effective lack of quantum coherence, and it (rather than inelastic scattering) usually provides the bound on the carrier phase coherence length $L_{\varphi}$ in experiments.

In clean semiconductors, where impurity scattering is minimal, $l$ can be tens of microns. This is not to say that scattering occurs only after the carriers have traveled a distance of several microns, but that, on average, the carrier goes about a mean free path length before the momentum direction is reversed. In fact there are a large number of "soft" scattering events along the way that deflect the carrier direction slightly, but do not prevent the carrier from continuing along nearly the same direction [10]. The value of $l$ is inferred from the measured conductance, so it is heavily weighted by carriers whose momenta are reversed (backscattered so that they do not reach the sensing contacts), and $l$ is largely oblivious to small angle scattering. Even so, the small angle scattering occurs only over comparatively large distances $(300-500 \mathrm{~nm})$ with respect to metals, and the lack of scattering has several remarkable consequences. 
For example, if two large area conductors are joined by a small "point contact", the conductance between the two large conductors exhibits steps as a function of the width of the contact [11]. The devices comprise a high mobility two-dimensional electron gas (2DEG) with either two patterned gate electrodes that nearly meet at a point on the surface [12] or a single continuous sheet of gate metal that covers the entire device region but is recessed from the carriers in regions where carriers are to remain [13]. Schematic drawings of the device structure
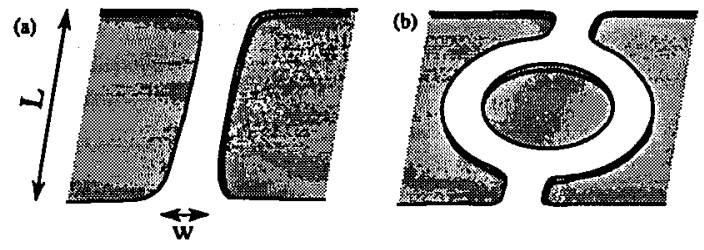

(c)

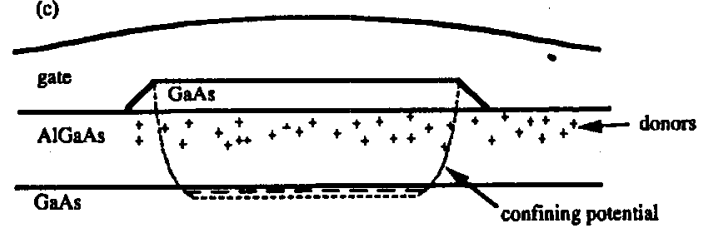

Fig. 1. (a) Schematic drawing of a point contact of length $L$ and width $w$ joining two large area conductors. (b) A similar structure in the shape of a loop interferometer. (c) Details of the crystallographically etched heterostructure and "global" gate used to implement the devices such as (a) and (b).

appear in Figs. 1a and 1c, where for the first scheme the shaded regions might depict the gate metal and in the second scheme they depict etched regions that allow the gate metal (Fig. 1c) greater capacitive influence on the 2DEG. When the gates are biased to deplete the carriers beneath them, they pinch the point contact reducing both its depth and width electrostatically. The steps arise in $G\left(V_{\mathrm{g}}\right)$ because the individual modes (quantized transverse momentum) are resolved, and because the individual modes propagate adiabatically $\left(W_{m}=1\right)$ through the point contact. Each mode contributes independently to the total conductance. The steps in conductance arise as the new modes pop through the Fermi surface one at a time so long as their separation exceeds $k_{\mathrm{B}} T$. Experiments in weak $[13,14]$ and strong [15] magnetic fields obtained very non-classical behavior as well in the magnetoresistance, which has been attributed to extraordinary momentum conservation lengths [16]. As the magnetic field $B$ increases and $\hbar \omega_{\mathrm{c}} \gg 1$ (where $\omega_{\mathrm{c}}=e B / m$ is the cyclotron frequency), the transport crosses over to a "globally adiabatic". regime [17] where the momentum conservation length is many millimeters [18].

So far only singly connected structures have been discussed. These have the generic form shown in Fig. 1a, where two wide conductors are connected by a narrow port of length $L$ and width $w$. Another somewhat more versatile tool for studying quantum interference is a loop structure shown schematically in Fig. 1b. The structure is reminiscent of optical two-slit interferometers [19], Aharonov-Bohm 
effect experiments [20] and the cylindrical sample geometries used to explore magnetic flux quantization and order parameter rigidity in superconductors and metals at low temperatures [21]. Experiments on clean structures of this geometry and its variations are the topic of the following discussion.
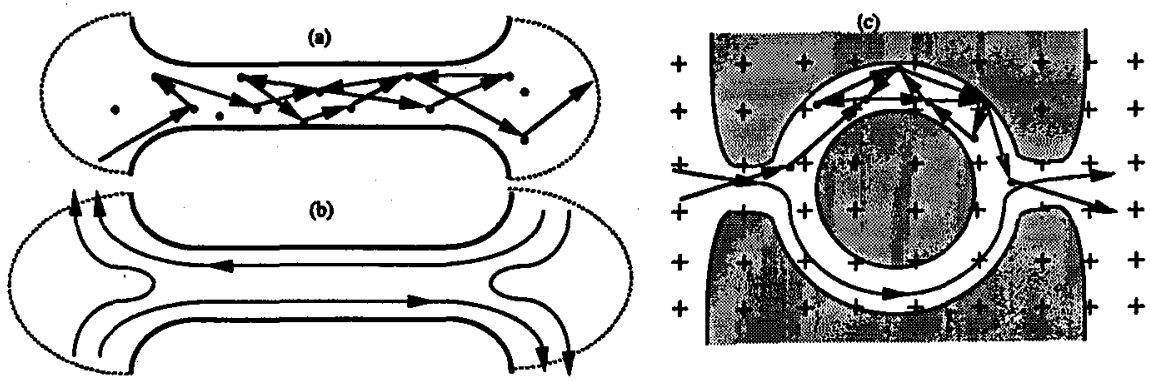

Fig. 2. (a) Illustration of random walk trajectory (arrows) scattered by impurities (dots) in a disordered conductor (heavy lines). (b) Illustration of adiabatic trajectories (including reflected trajectories in the wide areas) in a ballistic conductor. In both (a) and (b), the dotted line represents the phase coherent region. (c) Schematic of the interference experiment with loop sample that might support diffusive trajectories (upper half) or ballistic trajectories (lower half). The loop is surrounded by insulating (shaded regions) and a uniform magnetic field (pluses) penetrates the whole sample area.

The difference between the two regimes is portrayed in Figs. 2a and $b$. The carriers in the diffusive conductor (a) ricochet among the impurities, but in a ballistic conductor they pass through the constriction adiabatically (joining on continuously with transport states in the wide regions of the conductor [17, 22]). The two systems have very different conductances. The diffusive response is governed by details of the scattering of a large number of trajectories among the impurity sites. This leads to a random component to the conductance, which can be predicted only in the statistical sense. In fact, the details of the conductance are sensitive to the positions of every impurity, and moving so much as a single one completely "rewrites" the interference pattern [23]. In the ballistic conductor, on the other hand, the trajectories follow the walls of the conductor, and in principle, they can be governed by the architect of the conductor. Its conductance can be predicted in considerable detail through precise modeling [24].

One useful question to answer is: can one implement such a predictable solid-state interferometer? An affirmative answer to the question above appears to be given in the data of Figs. 3 and 4 and in data from other experiments at high magnetic field [15]. Figure 3 contains $G\left(V_{g}\right)$ for a loop sample (average diameter $L=1.6 \mu \mathrm{m}$ ) made from a high mobility $\mathrm{GaAs} / \mathrm{Al}_{0.3} \mathrm{Ga}_{0.7} \mathrm{As}$ heterostructure [25] and two traces $G(B)$ at fixed $V_{\mathrm{g}}$ from the same sample. The loops comprise crystallographically etched conductors forming a ring with annular width $w_{\mathrm{r}}=0.4 \mu \mathrm{m}$ connected to wide areas by ports of width $w_{\mathrm{p}}=0.3 \mu \mathrm{m}$ and length $L_{\mathrm{p}}=0.5 \mu \mathrm{m}$. It is important to notice that the ports alone are too long to exhibit ballistic conductance in other device manufacturing schemes [26], which appear to have been 


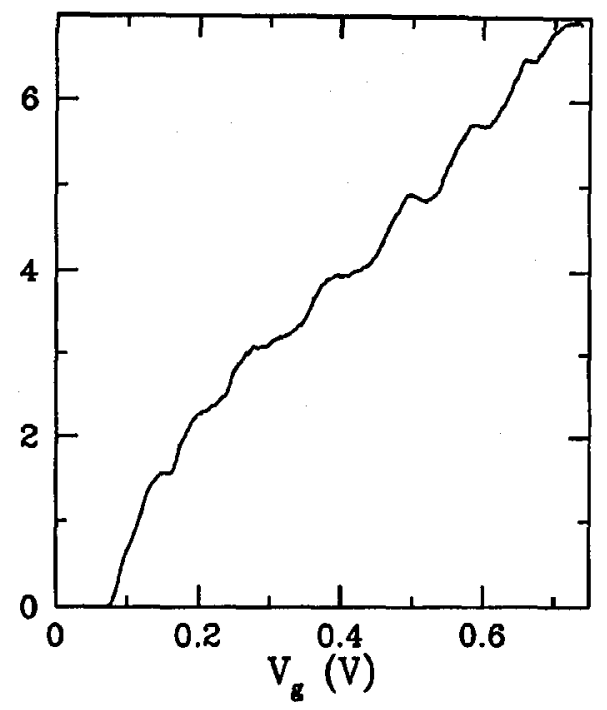

Fig. 3. $G\left(V_{\mathrm{g}}\right)$ measured at $T=4.2 \mathrm{~K}$ for a loop structure. The abscissa has been shifted to account for the difference in threshold voltage between the $4.2 \mathrm{~K}$ experiment and the $0.02 \mathrm{~K}$ experiment (adapted from Ref. [25]).

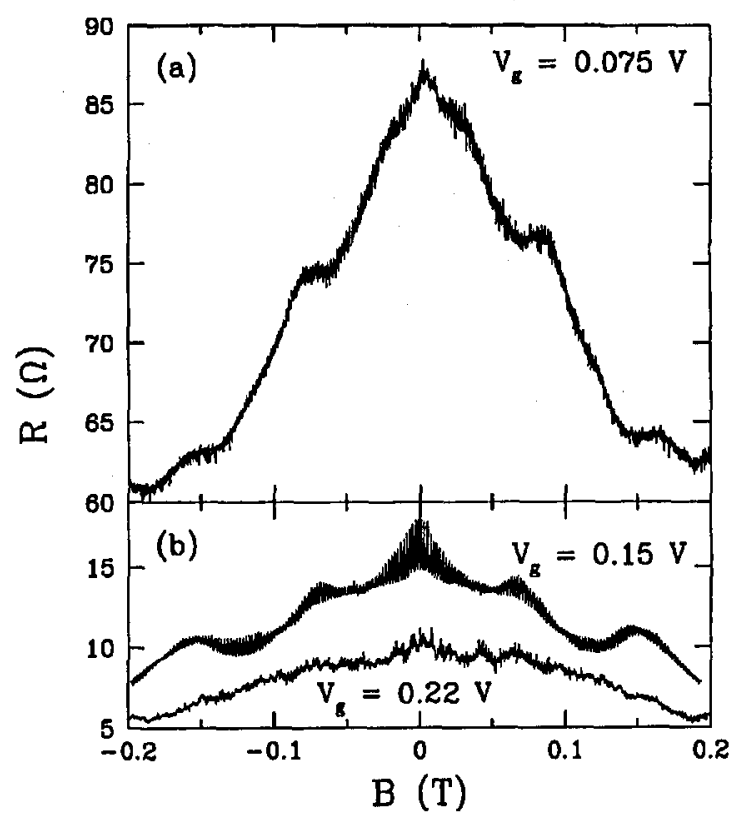

Fig. 4. Magnetoresistance of a loop measured at three different gate voltages (refer to Fig. 3) at $T=0.02 \mathrm{~K}$ in a carefully shielded environment (adapted from Ref. [25]). 
limited by the forward scattering length of a few hundred $\mathrm{nm}$. Nevertheless $G\left(V_{\mathrm{g}}\right)$ contains steps typical of momentum mode preservation, i.e. ballistic transport. The reason for the qualitatively different results from these devices is not clear. It might be related to deformation potentials caused by the gate metals [27], but this question awaits more serious study.

The magnetoresistance traces in Fig. 4b are (top) from a terrace in the $G\left(V_{\mathrm{g}}\right)$ staircase at $V_{\mathrm{g}}=0.15 \mathrm{~V}$ and (bottom) from nearby $V_{\mathrm{g}}=0.22 \mathrm{~V}$. The random character is evident in the lower trace and there is apparent lack of randomness in the upper trace. Both magnetoresistance traces comprise conductance components

$$
G(B)=G_{\text {class }}+\sum_{j}\left[G_{j}(B) \cos \left(\frac{2 \pi \Phi}{h / e}\right)+\alpha_{j}(B)\right] .
$$

Here $\Phi$ is the magnetic flux enclosed by the loop and $\alpha_{j}(B)$ is a random function of $B$ that accounts the random amounts of flux enclosed by different trajectories through the loop. The periodic modulation of the $A B$ oscillation amplitude in the $V_{\mathrm{g}}=0.15 \mathrm{~V}$ data arises from trapping of cyclotron orbits on the loop when the cyclotron orbit size of a particular mode matches the size of the loop [28]. The effect of the orbit trapping is of course to increase the average resistance and to change the probability of interference $P$ for the trajectories.

For the $V_{\mathrm{g}}=0.22 \mathrm{~V}$ trace $G_{1}(B)$ is rather small and randomly fluctuating in $B$. In the $V_{\mathrm{g}}=0.15 \mathrm{~V}$, however, the amplitude oscillates more-or-less periodically in $B$, and it is a factor of about 5 larger. Both the large amplitude and the regular pattern of "beating" of the $A B$ oscillation amplitude are reminiscent of very simple interference experiments such as might be seen in two-slit optics. It is very difficult to surmise that it results from random scattering of the carriers by impurities. The degree of randomness in the $A B$ oscillation amplitude $G_{1}(B)$ has been correlated to the step structure in $G\left(V_{\mathrm{g}}\right)$ with some success [29].

Although rather good modeling of the interference pattern is possible in open geometries [15], there has yet to be any firm correlation between the interference data from loops with device model calculations. In fact some device model calculations [30] tend to preclude the possibility of ballistic transport on the size scale $(\pi L=5 \mu \mathrm{m})$ of the devices used to obtain the data. Given the dramatic difference in the quality of the interference signatures from the two regimes of device operation, we are unable to draw any conclusion except that the ballistic motion of the carriers is dominating at $V_{\mathrm{g}}=0.15 \mathrm{~V}$.

Figure 4a contains resistance measured below the threshold for ballistic conductance where not even a single channel propagates without tunneling. It is conceivable that the situation might mimic the double-barrier tunneling configuration, which is being modeled with enthusiasm to infer the behavior of interacting strongly electrons (Luttinger liquid). This is not deep in the tunneling regime, but the sample resistance is as high as necessary to observe the predicted Luttinger effects. For very low density electron gases, one expects that the interactions to change the effective electrical charge of the carriers [31]. So far the experiment has not confirmed this prediction, but the predicted effect is weak and the experiment still rather crude. 
The same kind of experiments can be performed at high magnetic field, where $\hbar \omega_{\mathrm{c}} \ll 1$ and the transport is dominated by edge states that maintain their momentum for macroscopic distances [16]. The high field magnetoconductance of several ballistic conductors has pointed toward even greater opportunity to make predictable interferometers - at least in an open geometry [15]. In the loop samples, $G(B)$ from coupled interferometers allowed measurement of $L_{\varphi}$ through analysis of the $A B$ oscillation amplitude. The amplitude is proportional to the probability to interfere and the probability to retain phase coherence, i.e.

$$
G_{n}(B) \propto \exp \left(-\frac{n w^{2}}{4 r_{B}^{2}}\right) \exp \left(-\frac{n L}{L_{\varphi}}\right)=P_{n} Z_{n}
$$

where $r_{B}^{2}=h / e \pi B$ is related to the confinement of the wave function by the magnetic field. The first factor decays at high field and the second factor has been presumed widely to be a constant independent of $B$ [32]. By fitting (at very high field where it dominates) and scaling out the scattering probability $P_{n}$ we have obtained the bare phase coherence factor

$$
Z_{n}(B)=\frac{G_{n}(B)}{\exp (-0.49 n B)}
$$

which is plotted in Fig. $5 \mathrm{a}$ for $n=1$ and $n=2$. In addition to the large fluctuations

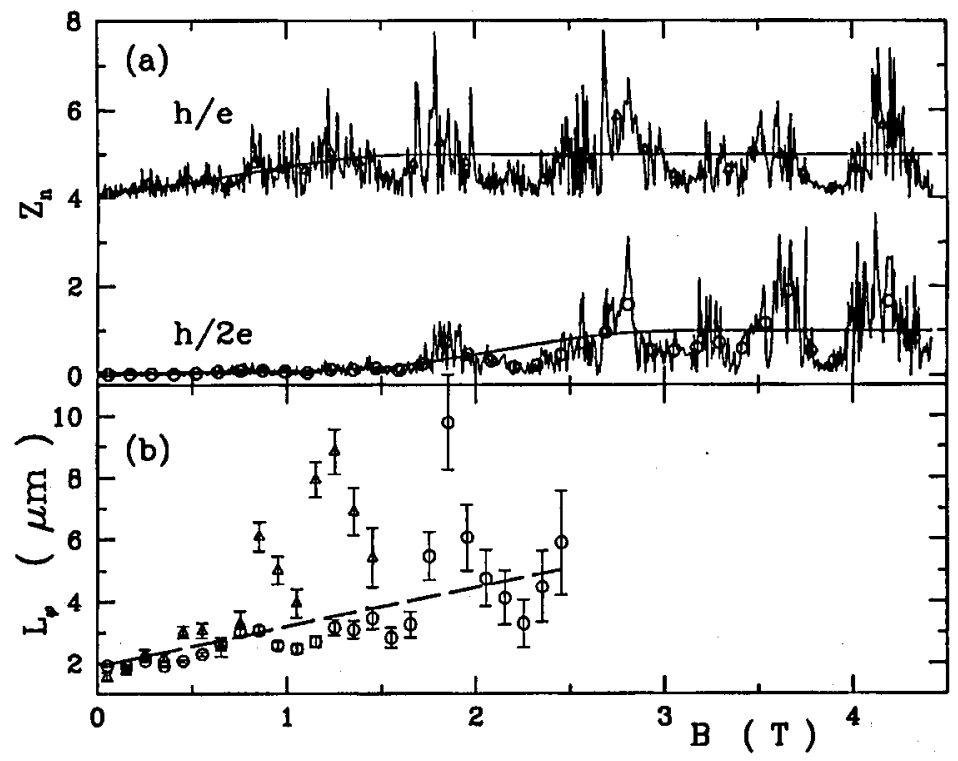

Fig. 5. (a) Amplitude functions $Z_{1}(B)$ and $Z_{2}(B)$ after normalizing them by removing (in a crude approximation) the scattering probability $P_{n}=\mathrm{e}^{-0.49 n B}$. (b) Inferred values of $L_{\varphi}$ obtained by averaging $G_{1}(B)$ and $G_{2}(B)$ (symbols in (a)) and inverting the formula in the text (adapted from Ref. [33]). 
that appear with the Shubnikov-de Haas peaks the average amplitudes clearly are increasing with increasing $B$ at low field. We can explain this increase with an increase in $L_{\varphi}$, and the smooth curves in the figure are fits to both of the amplitudes with a single linear function $L_{\varphi}(B)=2+1.3 B$, where $L_{\varphi}$ is measured in $\mu \mathrm{m}$ and $B$ is measured in tesla. The lower panel in the figure contains the $Z_{n}$ inverted to calculate the inferred values of $L_{\varphi}$. Error bars are estimated as well and of course they become large when $L_{\varphi} \gg \pi L$ and the exponential approaches unity [33].

While tantalizing, the results have not completely confirmed the possibility of predictable ballistic interference devices. Dramatic correlations between gate voltage and oscillation amplitude have been observed previously in samples where there was no clear mode counting staircase in $G\left(V_{\mathrm{g}}\right)$ [34]. Better controlled experiments and detailed theoretical modeling are needed to make more progress in this field.

\section{Acknowledgment}

It is a pleasure to acknowledge valuable collaborations and conversations with B. Altshuler, H. Baranger, M. Büttiker, J. Davies, M. Fisher, C. Ford, A. Fowler, W.-X. Gao, M. Hong, Y. Imry, K. Ismail, K. Lee, J. Liu and R. Webb.

\section{References}

[1] See for example several articles in Mesoscopic Phenomena in Solids, Eds. B.L. Altshuler, P.A. Lee, R.A. Webb, Elsevier, Amsterdam 1991, and references cited there.

[2] S. Chakravarty, A. Schmid, Phys. Rep. 140, 193 (1986) and references cited there.

[3] S. Washburn, R.A. Webb, Rep. Prog. Phys. 55, 1311 (1992) and references cited there.

[4] See for example, Magnetoresistance in Metals, Ed. A.B. Pippard, Cambridge University Press, Cambridge 1989; or V.S. Tsoi, J. Bass, P. Wyder, Adv. Phys. 41, 365 (1992) for more detail.

[5] C.W.J. Beenakker, H. van Houten, in: Solid State Physics, Vol. 44, Eds. H. Ehrenreich, D. Turnbull, Academic, New York 1991, p. 1; G. Timp, in Ref. [1], p. 273.

[6] P.F. Maldague, Phys. Rev. B 23, 1719 (1981); B.L. Altshuler, Pis'ma Zh. Eksp. Teor. Fiz. 41, 530 (1985) [JETP Lett. 41, 648 (1985)]; P.A. Lee, A.D. Stone, Phys. Rev. Lett. 55, 1622 (1985).

[7] B.L. Altshuler, B.I. Shklovskii, Zh. Eksp. Teor. Fiz. 91, 220 (1986) [JETP 64, 127 (1986)].

[8] A.D. Stone, Phys. Rev. B 39, 10376 (1989).

[9] B.L. Altshuler, D.E. Khmelnitskii, Pis'ma Zh. Eksp. Teor. Fiz. 42, 291 (1985) [JETP Lett. 42, 359 (1985)].

[10] S. Das Sarma, F. Stern, Phys. Rev. B 32, 8442 (1985). 
[11] Y.Imry, in: Directions in Condensed Matter Physics, Eds. G. Grinstein, E. Mazenko, World Scientific, Singapore 1986, p. 100; D.A. Wharam, T.J. Thornton, R. Newbury, M. Pepper, H. Ahmed, J.E.F. Frost, D.G. Hasko, D.C. Peacock, D.A. Ritchie, G.A.C. Jones, J. Phys. C 21, L209 (1988); B. van Wees, L.P. Kouwenhoven, E.M.M. Willems, C.J.P.M. Harmans, J.E. Mooij, H. van Houten, C.W.J. Beenakker, J.G. Williamson, C.T. Foxon, Phys. Rev. B 43, 12431 (1991); G. Timp, in: Nanostructured Systems, Ed. M.A. Reed, Academic, New York 1990, p. 113.

[12] T.J. Thornton, M. Pepper, H. Ahmed, G.J. Davies, D. Andrews, Phys. Rev. Lett. 56, 1198 (1987).

[13] C.J.B. Ford, S. Washburn, M. Büttiker, C.M. Knoedler, J.M. Hong, Phys. Rev. Lett. 62, 2724 (1989).

[14] G. Timp, H.U. Baranger, P. deVegvar, J.E. Cunningham, R.E. Howard, R. Behringer, P.M. Mankiewich, Phys. Rev. Lett. 60, 2081 (1988); H. van Houten, C.W.J. Beenakker, J.G. Williamson, M.E.I. Broekhart, P.H.M. van Loosdrecht, B. van Wees, J.E. Mooij, C.T. Foxon, J.J. Harris, Phys. Rev. B 39, 8556 (1989).

[15] B.J. van Wees et al. (see [11]); B. van Wees, L.P. Kouwenhoven, C.J.P.M. Harmans, J.G. Williamson, C.E. Timmering, M.E.I. Broekhart, C.T. Foxon, J.J. Harris, Phys. Rev. Lett. 62, 2523 (1989).

[16] S. Komiyama, H. Hirai, M. Ohsawa, Y. Matsuda, Phys. Rev. B 45, 11085 (1992) and references cited there; M. Büttiker, in: Nanostructured Systems, Ed. M.A. Reed, Academic, Boston 1992, p. 191; R.J. Haug, Semicond. Sci. Technol. 8, 131 (1993) and references cited there.

[17] L.I. Glazman, M. Jonson, Phys. Rev. B 41, 10686 (1990).

[18] B.L. Alphenaar, P.L. McEuen, R.G. Wheeler, R.N. Sacks, Phys. Rev. Lett. 64, 677 (1990); P.L. McEuen, A. Szafer, C.A. Richter, B.W. Alphenaar, J.K. Jain, A.D. Stone, R.G. Wheeler, R.N. Sacks, Phys. Rev. Lett. 64, 2062 (1990); S. Komiyama et al. (see [16]).

[19] R.P. Feynman, R.B. Leighton, M. Sands, The Feynman Lectures on Physics, Vol. I and III, Addison-Wesley, Reading 1965, pp. I-15-5, III-3-5.

[20] A. Tonomura, M.A. Peshkin, The Aharonov-Bohm Effect, Springer, Heidelberg 1989; S. Olariu, I.I. Pospescu, Rev. Mod. Phys. 57, 339 (1985).

[21] A.G. Aronov, Yu.V. Sharvin, Rev. Mod. Phys. 59, 755 (1987).

[22] L.I. Glazman, G.B. Lesovik, D.E. Khmel'nitskii, R.I. Shektar, Pis'ma Zh. Eksp. Teor. Fiz. 48, 218 (1988) [JETP Lett. 48, 238 (1988)].

[23] B.L. Altshuler, B.Z. Spivak, Pis'ma Zh. Eksp. Teor. Fiz. 42, 363 (1985) [JETP Lett. 42, 447 (1985)].

[24] H.U. Baranger, R.A. Jalabert, A.D. Stone, Chaos 3, 665 (1993), and references cited there.

[25] K. Ismail, S. Washburn, K.Y. Lee, Appl. Phys. Lett. 59, 1998 (1991); S. Washburn, K. Ismail, K.Y. Lee, in: Quantum Effect Physics Electronics and Applications, Eds. K. Ismail, H.I. Smith, T. Ikoma, Hilger, Bristol 1992, p. 153; K.Y. Lee, D. Kern, K. Ismail, S. Washburn, J. Vac. Sci. Technol. B 9, 2834 (1991).

[26] G. Timp (see [11]).

[27] J.H. Davies, I.A. Larkin, to be published.

[28] J. Liu, K. Ismail, K.Y. Lee, J.M. Hong, S. Washburn, Phys. Rev. B 47, 13039 (1993).

[29] J. Liu, W.X. Gao, K. Ismail, K.Y. Lee, J.M. Hong, S. Washburn, Phys. Rev. B 48, 15148 (1993). 
[30] J.A. Nixon, J.H. Davies, H.U. Baranger, Phys. Rev. B 43, 12638 (1991).

[31] C.L. Kane, M.P.A. Fisher, Phys. Rev. Lett. 68, 1220 (1992).

[32] See however, M. Büttiker, in Ref. [16]; T. Martin, S. Feng, Phys. Rev. Lett. 64, 1971 (1990), Phys. Rev. B 44, 9084 (1991) and private communication; G. Fasol, Solid State Commun. 84, 77 (1992); H. Hirai, S. Komiyama, K. Nakamura, F. Nihey, Physica 184, 34 (1993).

[33] J. Liu, W.X. Gao, K. Ismail, K.Y. Lee, J.M. Hong, S. Washburn, J. Low. Temp. Phys., to appear and Phys. Rev. B, to appear.

[34] C.J.B. Ford, A.B. Fowler, J.M. Hong, C.M. Knoedler, S.E. Laux, J.J. Wainer, S. Washburn, Surf. Sci. 229, 307 (1990). 